University of Windsor

Scholarship at UWindsor

2005

\title{
Intramolecular Pauson-Khand reactions of cycloheptynedicobalt complexes
}

\author{
Ahmed B. Mohamed \\ James R. Green \\ University of Windsor \\ Jason Masuda
}

Follow this and additional works at: https://scholar.uwindsor.ca/chemistrybiochemistrypub

Part of the Chemistry Commons

\section{Recommended Citation}

Mohamed, Ahmed B.; Green, James R.; and Masuda, Jason. (2005). Intramolecular Pauson-Khand reactions of cycloheptynedicobalt complexes. Synlett (10), 1543-1546.

https://scholar.uwindsor.ca/chemistrybiochemistrypub/16

This Article is brought to you for free and open access by the Department of Chemistry and Biochemistry at Scholarship at UWindsor. It has been accepted for inclusion in Chemistry and Biochemistry Publications by an authorized administrator of Scholarship at UWindsor. For more information, please contact scholarship@uwindsor.ca. 


\title{
Intramolecular Pauson-Khand reactions of cycloheptynedicobalt complexes
}

\author{
Ahmed B. Mohamed, James R. Green*, Jason Masuda \\ Department of Chemistry and Biochemistry, University of Windsor, Windsor, Ontario, N9B 3P4, Canada \\ Fax: (519)-973-7098 \\ E-mail: jgreen@uwindsor.ca \\ Received: The date will be inserted once the manuscript is accepted.
}

Abstract: Cycloheptyne- $\mathrm{Co}_{2}(\mathrm{CO})_{6}$ complexes bearing alkenes tethered by oxygen, sulphur, and nitrogen atoms undergo PausonKhand reactions to afford tricyclic compounds containing a fused 7,5- ring system.

Key words: Pauson-Khand reactions, alkynes, complexes, transition metals, Nicholas reactions

The fused 7,5- ring system an is extremely common framework within natural products, particular those of the terpene class. The guaiane, daucane, and lactarane sesquiterpenoids are only the most obvious of these systems, as many other sesquiterpenoids ${ }^{1}$ and diterpenoids contain this structural unit. While traditional synthetic methods predominantly rely upon ring expansion protocol for access to the cycloheptane portion of these systems, ${ }^{2}$ approaches based on direct cycloheptane synthesis are of increasing importance. ${ }^{3}$

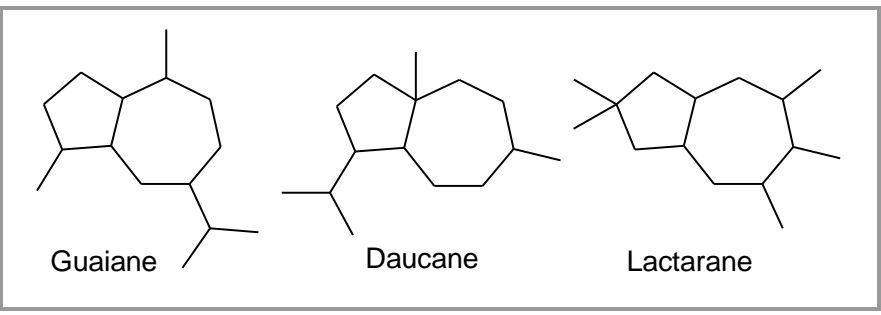

Figure 1 Guaiane, daucane, and lactarane sesquiterpenoids

It has been demonstrated our group ${ }^{4}$ and other groups ${ }^{5}$ that cycloheptynedicobalt complexes are both stable and readily accessible, predominantly by Nicholas reaction chemistry. Due to the strain associated with cyclic alkynes, the $\mathrm{Co}_{2}(\mathrm{CO})_{6}$ unit is central to the existence of the ring system. In addition, alkyne- $\mathrm{Co}_{2}(\mathrm{CO})_{6}$ complexes are among the most widely employed precursors to fivemembered ring construction by Pauson-Khand reactions, ${ }^{6,7}$ despite recent important advances in catalytic methods and the use of other metal complexes. ${ }^{6 a, d}$ As a result, it was our belief that the investigation of PausonKhand reactions on cycloheptynedicobalt complexes would be of importance for the synthesis of 7,5- ring systems. As early work in our group indicated that intermolecular Pauson-Khand reactions of cycloheptenyne- $\mathrm{Co}_{2}(\mathrm{CO})_{6}$ complexes succeeded with a quite restricted number of alkenes, ${ }^{8}$ we concluded that intramolecular versions of the reaction were prudent. It was our intent to incorporate an alkene function with each of oxygen, sulphur, and nitrogen tethers to the alkynedicobalt unit, with particular interest in oxygen and sulphur tethers due to their capacity for reductive cleavage in the products. ${ }^{9,10}$ An analogous approach employing oxygen tethered alkenes has seen success in a limited number of instances for 8,5- and 9,5- ring systems. ${ }^{11,5 \mathrm{~b}, 5 \mathrm{f}}$ While this work was nearing completion, the preparation of 5,7,5- and 5,8,5- systems by way of intramolecular Pauson-Khand reactions of 7- and 8- membered ring cyclic ether alkyne- $\mathrm{Co}_{2}(\mathrm{CO})_{6}$ complexes with carbonbased alkene tethers was reported by the group of Shea. ${ }^{12}$

We chose as our starting point homoallyl propargyl acetate 1a, which may be obtained by ring closing metathesis of the appropriate acyclic diene, ${ }^{46,5 b}$ and the corresponding cycloheptyne complex $\mathbf{1 b}$, which was prepared quantitatively by Raney ${ }^{\circledR}$ nickel reduction of 1a. ${ }^{13}$ Complex 1a could be converted into allyl ether 2a $(75 \%$ yield) and allyl thioether $\mathbf{2 b}\left(58 \%\right.$ yield) by $\mathrm{BF}_{3}-\mathrm{OEt}_{2}$ ( 5 equiv) with allyl alcohol or allylthiol, respectively. ${ }^{14}$ In these cases, the reactions had to be performed in the presence of excess alcohol/thiol (10 equiv) in order to effectively compete with elimination of acetic acid from 1a. In the case of acetate $\mathbf{1 b}$, the analogous elimination process was less of a competitive one, such that $\mathbf{1 b}$ could be converted into allyl alcohols $\mathbf{2 c}$ ( $84 \%$ yield) and 2 d (84\% yield), and allyl thioether $2 \mathbf{e}(84 \%)$ by treatment with $\mathrm{BF}_{3}-\mathrm{OEt}_{2}$ and allyl alcohol, 2-methyl-2-propen-1ol, and allylthiol, respectively. In addition, homoallyl alcohol $2 f$ ( $92 \%$ yield) could be obtained by the analogous reaction with 1-buten-4-ol.

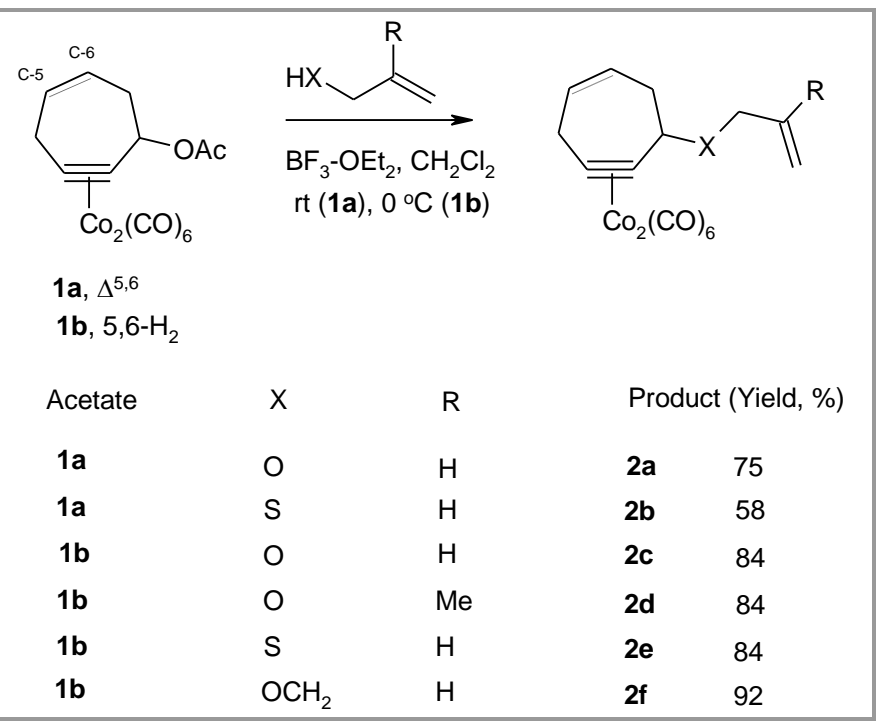

Scheme 1 Preparation of oxygen- and sulphur tethered enyne$\mathrm{Co}_{2}(\mathrm{CO})_{6}$ complexes

Our desire to include a nitrogen tethered substrate initially met with failure. Subjecting acetate $\mathbf{1 b}$ to allylamine or N-allylacetamide under protic or Lewis acidic condi- 
tion gave no amine incorporation with allylamine and at best trace amounts of amide incorporation with $\mathrm{N}$ allylacetamide (2g). Attempts to isolate cation $\mathbf{3}$ by treatment of $\mathbf{1 b}$ or alcohol $\mathbf{1 c}$ with tetrafluoroboric acid were similarly unsuccessful. Fortunately, the use of $\mathrm{HBF}_{4}$ with excess dimethyl sulphide rapidly gave a precipitate consistent with the formation of sulphonium salt 4. ${ }^{15}$ Removal of solvent from 4 under vacuum and subsequent addition of allylamine and diisopropylethylamine afforded $\mathbf{2 h}$, which was in turn immediately subjected to $p$-toluenesulphonic anhydride, giving $\mathbf{2 i}$ in $62 \%$ yield (from 1b). While $\mathrm{N}$ - acetyl functions are infrequently used as tethers for intramolecular Pauson-Khand reactions, ${ }^{16}$ we desired an additional example of this $\mathrm{N}$ substitution sequence; therefore, employing acetic anhydride in place of $p$-toluenesulphonic anhydride in reaction with unpurified $\mathbf{2 h}$ afforded $\mathbf{2 g}$ in $73 \%$ yield (from 1b).

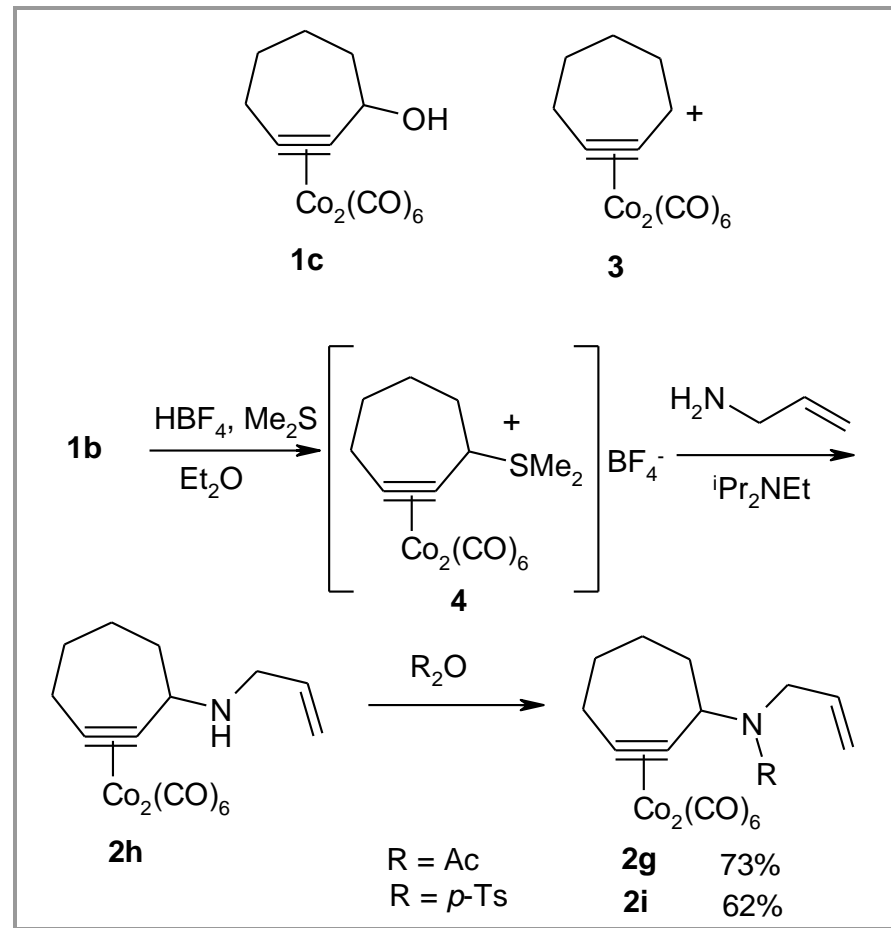

Scheme 2 Preparation of nitrogen tethered enyne- $\mathrm{Co}_{2}(\mathrm{CO})_{6}$ complexes

With several substrates in hand, attention was turned to the Pauson-Khand reactions of $\mathbf{2 a - f}$, and $\mathbf{2 i}$. We chose $\mathbf{2 c}$ for investigation of the reaction under several sets of conditions. The Sugihara amine conditions (3.5 equiv $\mathrm{CyNH}_{2}, \mathrm{ClCH}_{2} \mathrm{CH}_{2} \mathrm{Cl}$, reflux ${ }^{17}$ indeed gave PausonKhand product 5c in $65 \%$ yield, as a 5.2:1 mixture of diastereomers favouring the isomer containing methine hydrogen atoms in a cis- relationship (cis- 5c), along with a smaller amount of Pauson-Khand/allylic reduction product $^{17 a}$ 6c (13\%). Attempted use of the Sugihara sulphide conditions (BuSMe, $\mathrm{ClCH}_{2} \mathrm{CH}_{2} \mathrm{Cl}$, reflux) ${ }^{18}$ eliminated the byproduct $\mathbf{6 c}$, but at the expense of $\mathbf{5 c}(51 \%$, 2.0:1 cis:trans). In addition, $\mathrm{Me}_{3} \mathrm{NO}$ (49\% yield of $\mathbf{5 c}$, 1.2:1 cis:trans) and refluxing toluene (20\% yield of $\mathbf{5 c}$, 1.1:1 cis:trans) were judged inferior to the Sugihara amine conditions.
The Sugihara amine conditions were therefore explored with the other substrates. The corresponding allyl/homoallyl ether complex 2a cyclized to form 5a in excellent yield $(89 \%)$ and good diastereoselectivity (cis:trans $=10: 1)$. Both thioallyl complexes $\mathbf{2 b}$ and $\mathbf{2 e}$ cyclized successfully to give $\mathbf{5 b}(65 \%$ yield, cis:trans $=$ $3.8: 1)$ and 5e $(66 \%$ yield, cis:trans $=2.0: 1)$, respectively, although the diastereoselectivities were lower than the oxygen tethered analogues. Homoallyl ether complex $2 \mathbf{f}$ similarly underwent reaction to give $\mathbf{5 f}$ (73\% yield, cis:trans $=2.0: 1){ }^{19}$

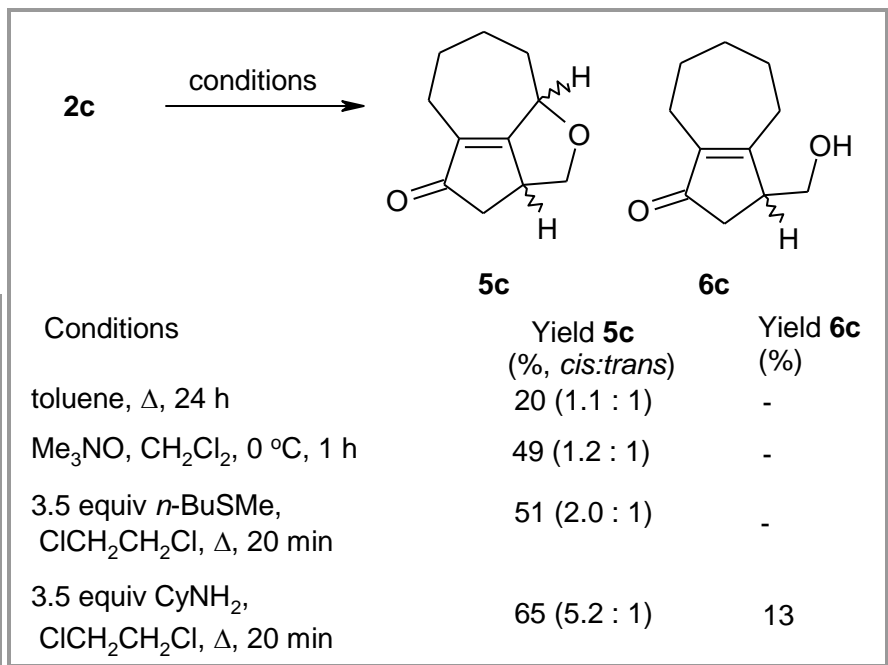

Scheme 3 Pauson-Khand reaction of $2 \mathbf{c}$ under various conditions

In contrast to the above cases, both methallyl ether $\mathbf{2 d}$ and $\mathrm{N}$-allyl tosylate complex $\mathbf{2} \mathbf{i}$ failed to give PausonKhand reaction products in synthetically useful yields under the Sugihara amine conditions, although a small amount of Pauson-Khand/allylic reduction product $6 \mathbf{6}$ (14\% yield) was obtained from 2d. Therefore, several other sets of reaction protocols were examined with these substrates. Fortunately, methallyl ether complex 2d underwent cyclization successfully under $\mathrm{Me}_{3} \mathrm{NO}$ conditions $\left(0{ }^{\circ} \mathrm{C}, 59 \%\right.$ yield, $66 \%$ yield based on recovered 2d) to give cis-5d exclusively. Sulphonamide tethered complex $2 \mathbf{i}$ cyclized successfully under the Sugihara sulphide conditions, to give $\mathbf{5 i}$ (55\% yield, cis:trans $=$ $1.2: 1)^{20}$

The stereochemical assignments were arrived at as follows. To our surprise, the ${ }^{1} \mathrm{H}$ NMR spectra of majority of 5a-f and 5i did not give nOe enhancements between the methine hydrogen atoms for either diastereomer. Conversely, the 2D-NOESY spectrum of $\beta$-methyl substituted $\mathbf{5 d}$ displayed a positive nOe interaction between the methine hydrogen and the methyl hydrogens, which was therefore indicative of a cis-relationship. The methine $\mathrm{H}$ atoms $\alpha$ - to the heteroatoms of $\mathbf{5 d}$ and the major diastereomers of the cycloheptane products $\mathbf{5 c}, \mathbf{5 f}$, and $\mathbf{5 i}$ also displayed very similar ${ }^{1} \mathrm{H}$ NMR vicinal coupling constants (dd, $\mathrm{J}=9.9-10.9,4.2-5.7$ ) which were not repeated in the minor diastereomers. Finally, a small amount of the minor isomer of 5e formed crystals suitable for X-ray diffraction studies, which indicated a trans- 
relationship between the two methines. The assignments for 5a-b were based on analogy and are supported by calculations on the proposed alkene coordination intermediate, the alkene insertion transition state (MMX, PCMODEL; MM3, CAChe ${ }^{\mathscr{Q}}$ ), and on 5a-b themselves $\left(\mathrm{PM} 3, \mathrm{CAChe}^{\Theta}\right)$.

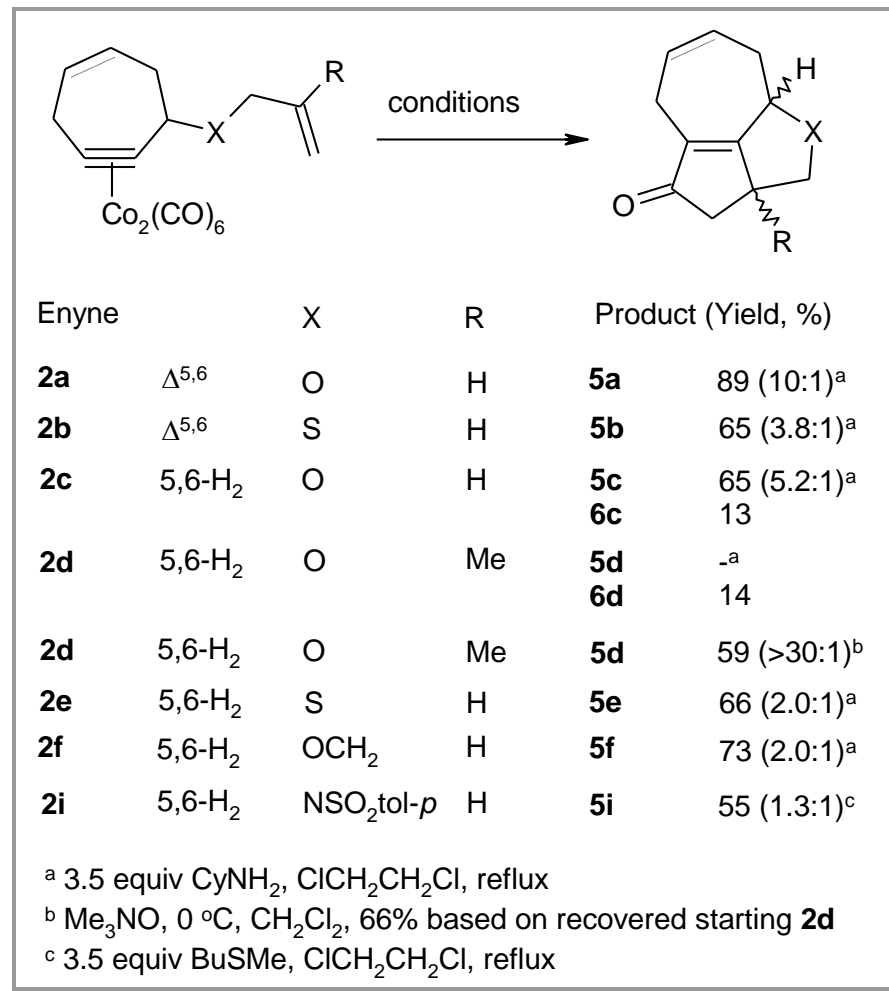

Scheme 4 Intramolecular Pauson-Khand reactions of cycloheptyne $\mathrm{Co}_{2}(\mathrm{CO})_{6}$ complexes

In summary, we have demonstrated the ability of oxygen-, sulphur-, and nitrogen tethers to enable PausonKhand reactions of cycloheptyne- $\mathrm{Co}_{2}(\mathrm{CO})_{6}$ complexes, affording tricyclic systems containing a fused 7,5- structural unit. As the resultant enone $\mathrm{C}=\mathrm{C}$ bond is mutual to the 7- and 5- membered rings, we view the chemistry as complementary to the work of the Moyano/Pericàs group and the group of Pérez-Castells, ${ }^{9 a, 21}$ who have employed Pauson-Khand reactions of alkynes tethered to cycloheptenes through sulphur, and oxygen and nitrogen atoms, respectively, to give 5,7,5- systems with the alkene function solely in the 5- membered ring.

\section{Acknowledgment}

We are grateful for NSERC (Canada), the Canada Foundation for Innovation (CFI), and the Ontario Innovation Trust for support of this research. We wish to thank the Windsor Molecular Structure Centre (WINMOL) for crystallographic work on trans-5e.

\section{References}

(1) (a) Fraga, B. M. Nat. Prod. Rep. 2004, 21, 669. (b) Hanson, J. R. Nat. Prod. Rep. 2004, 21, 785.

(2) (a) Ho, T.-L. Carbocycle Construction in Terpene Synthesis; VCH: New York, 1988; Chapter 11; (b) Ewing, D. F. in Rodd's Chemistry of Carbon Compounds, 2nd edn., M.
Sainsbury, M., Ed., Elsevier, Amsterdam, 1994, vol. 2, 2nd Suppl., Pt. B, C, D, E, ch 8 a.

(3) For example, see; Molander, G. Acc. Chem. Res. 1998, 31, 603. (b) Harmata, M. Acc. Chem. Res. 2001, 34, 595. (c)

Rigby, J. H.; Pigge, F. C. Org. React. 1997, 51, 351.

(4) (a) Ding, Y.; Green, J. R. Synlett 2005, 271; (b) Lu, Y.; Green, J. R. Synlett 2001, 243; Green, J. R. Synlett, 2001, 353; (c) Patel, M. M.; Green, J. R. Chem. Commun. 1999, 509; (d) Green, J. R. Chem. Commun. 1998, 1751.

(5) (a) Tanino, K.; Onuki, K.; Asano, K.; Miyashita, M.; Nakamura, T.; Takahashi, Y.; Kuwajima, I. J. Am. Chem. Soc. 2003, 125, 1498; (b) Young, D. G. J.; Burlison, J. A.; Peters, U. J. Org. Chem. 2003, 68, 3494; (c) Tanino, K.; Kondo, F.; Shimizu, T.; Miyashita, M. Org. Lett. 2002, 4, 2217; (d) Tanino, K.; Shimizu, T.; Miyama, M.; Kuwajima, I. J. Am. Chem. Soc. 2000, 122, 6116; (e) Iwasawa, N.; Sakurada, F.; Iwamoto, M. Org. Lett., 2000, 2, 871; (f) Schreiber, S. L.; Sammakia, T.; Crowe, W. E. J. Am. Chem. Soc. 1986, 108, 3128.

(6) (a) Park, K. H.; Chung, Y. K. Synlett 2005, 545; (b) Alcaide, B.; Almendros, P. Eur. J. Org. Chem. 2004, 3377; (c) Blanco-Urgoiti, J.; Añorbe, L.; Pérez-Serrano, L.; Domínguez, G.; Pérez-Castells, J. Chem. Soc. Rev. 2004, 33, 32; (d) Gibson, S. E.; Stevenazzi, A. Angew. Chem., Int. Ed. Engl. 2003, 42, 1800; (e) Rivero, Marta R.; Adrio, J.; Carretero, J. C. Eur. J. Org. Chem. 2002, 2881. (f) Welker, M. E. Curr. Org. Chem. 2001, 5, 785; (g) Brummond, K. M.; Kent, J. L. Tetrahedron 2000, 56, 3263.

(7) See also: Bonaga, L. V. R.; Krafft, M. E. Tetrahedron 2004, 60, 9795.

(8) Patel, M. M., M.Sc. Thesis, University of Windsor, Windsor, ON, Canada, 1999.

(9) (a) Castro, J.; Moyano, A.; Pericàs, M. A.; Riera, A. J. Org. Chem. 1998, 63, 3346; (b) Tius, M. A.; Kerr, M. A. J. Am. Chem. Soc. 1992, 114, 5959.

(10) For general reviews on tethers in organic synthesis, see: (a) Cox, L. R.; Ley, S. V. In Templated Organic Synthesis; Diederich, F; Stang, P. J, Eds.; Wiley-VCH: Weinheim, 2000; Chapter 10; (b) Knapp, S. Chem. Soc. Rev. 1999, 28 , 61. (c) Gauthier, D. R., Jr.; Zandi, K. S.; Shea, K. J. Tetrahedron 1998, 54, 2289.

(11) Jamison, T. F.; Shambayati, S.; Crowe, W. E.; Schreiber, S. L. J. Am. Chem. Soc. 1997, 119, 4353.

(12) Quintal, M. M.; Closser, K. D.; Shea, K. M. Org. Lett. 2004, 6, 4949.

(13) Mohamed, A. B; Green, J. R. Chem. Commun. 2003, 2936.

(14) Selected compounds: (2c):IR (neat, NaCl) $v_{\max } 2932,2090$, 2047, $2020 \mathrm{~cm}^{-1} ;{ }^{1} \mathrm{H}$ NMR $\delta 5.98(\mathrm{~m}, 1 \mathrm{H}), 5.34(\mathrm{dd}, \mathrm{J}=$ $17.1,1.5,1 \mathrm{H}), 5.21(\mathrm{dd}, \mathrm{J}=10.4,1.5,1 \mathrm{H}), 4.48(\mathrm{dd}, \mathrm{J}=$ $10.4,4.2,1 \mathrm{H}), 4.35(\mathrm{dd}, \mathrm{J}=12.5,5.1,1 \mathrm{H}), 4.19(\mathrm{dd}, \mathrm{J}=$ $12.5,5.7,1 \mathrm{H}), 3.19$ (apparent dt, $\mathrm{J}=16.7,3.4,1 \mathrm{H}), 2.76$ $(\mathrm{m}, 1 \mathrm{H}), 2.19(\mathrm{~m}, 1 \mathrm{H}), 2.05(\mathrm{~m}, 1 \mathrm{H}), 1.97(\mathrm{~m}, 1 \mathrm{H}), 1.48-$ $1.65(\mathrm{~m}, 2 \mathrm{H}), 1.39(\mathrm{~m}, 1 \mathrm{H}) ;{ }^{13} \mathrm{C}$ NMR 203.9, 138.7, 121.0, 103.6, 102.5, 84.4, 74.2, 40.9, 39.4, 33.1, 29.7 ; MS m/e $380\left(\mathrm{M}-2 \mathrm{CO}^{+}\right)$; HRMS m/e for $\mathrm{C}_{16} \mathrm{H}_{14} \mathrm{Co}_{2} \mathrm{O}_{7}$ calcd. (M$2 \mathrm{CO}^{+}$) 379.9500, found 379.9466; (2e) IR (neat, $\mathrm{NaCl}$ ) $v_{\max }$ 2927, 2089, 2049, $2020 \mathrm{~cm}^{-1}$; ${ }^{1} \mathrm{H}$ NMR $\delta 5.88(\mathrm{~m}, 1 \mathrm{H})$, $5.15(\mathrm{~d}, \mathrm{~J}=17.7,1 \mathrm{H}), 5.12(\mathrm{~d}, \mathrm{~J}=12.0,1 \mathrm{H}), 3.89(\mathrm{dd}, \mathrm{J}=$ $14.2,4.2,1 \mathrm{H}), 3.29(\mathrm{~d}, \mathrm{~J}=7.5,2 \mathrm{H}), 3.12(\mathrm{dt}, \mathrm{J}=16.3,3.6$, $1 \mathrm{H}), 2.82(\mathrm{~m}, 1 \mathrm{H}), 2.27(\mathrm{~m}, 1 \mathrm{H}), 2.11(\mathrm{~m}, 1 \mathrm{H}), 1.95(\mathrm{~m}$, 1H), $1.68(\mathrm{~m}, 1 \mathrm{H}), 1.59(\mathrm{~m}, 1 \mathrm{H}), 1.45(\mathrm{~m}, 1 \mathrm{H}) ;{ }^{13} \mathrm{C} \mathrm{NMR}$ 200.0, 134.7, 116.9, 102.0, 99.9. 48.5, 38.0, 35.5, 35.0,

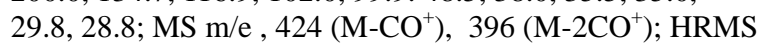
$\mathrm{m} / \mathrm{e}$ for $\mathrm{C}_{16} \mathrm{H}_{14} \mathrm{Co}_{2} \mathrm{O}_{6} \mathrm{~S}$ calcd. $\left(\mathrm{M}-2 \mathrm{CO}^{+}\right) 395.9271$, found 395.9295. (2i) IR (neat, KBr) $v_{\max } 3082,2928,2093,2051$, $2025 \mathrm{~cm}^{-1} ;{ }^{1} \mathrm{H}$ NMR $\delta 7.72(\mathrm{~d}, \mathrm{~J}=8.3,2 \mathrm{H}), 7.28(\mathrm{~d}, \mathrm{~J}=$ $8.3,2 \mathrm{H}), 5.76(\mathrm{~m}, 1 \mathrm{H}), 5.20(\mathrm{dd}, \mathrm{J}=17.2,1.3,1 \mathrm{H}), 5.07$ $(\mathrm{dd}, \mathrm{J}=10.2,1.3,1 \mathrm{H}), 4.96(\mathrm{dd}, \mathrm{J}=11.7,3.2,1 \mathrm{H}), 4.14-$ $4.26(\mathrm{~m}, 2 \mathrm{H}), 3.23(\mathrm{br} \mathrm{d}, \mathrm{J}=13.5,1 \mathrm{H}), 2.73(\mathrm{~m}, 1 \mathrm{H}), 2.43$ 
(s, 3H), $2.11(\mathrm{~m}, 1 \mathrm{H}), 1.92-2.04(\mathrm{~m}, 2 \mathrm{H}), 1.73(\mathrm{~m}, 1 \mathrm{H})$, 1.35-1.60 (m, 2H); ${ }^{13} \mathrm{C}$ NMR 199.1, 143.1, 138.6, 135.8, 129.5, 127.3, 117.3, 102.2, 96.9, 63.5, 46.2, 37.0, 35.6, 28.9, 21.5; MS m/e , $533\left(\mathrm{M}-2 \mathrm{CO}^{+}\right), 505\left(\mathrm{M}-3 \mathrm{CO}^{+}\right), 421$ $\left(\mathrm{M}-6 \mathrm{CO}^{+}\right.$) ; HRMS m/e for $\mathrm{C}_{23} \mathrm{H}_{21} \mathrm{Co}_{2} \mathrm{NO}_{8} \mathrm{~S}$ calcd. (M$3 \mathrm{CO}^{+}$) 504.9804, found 504.9828.

(15) El-Amouri, H.; Gruselle, M.; Jaouen, G.; Daran, J. C.; J. Vaissermann, J.; Gruselle, M. Inorg. Chem. 1990, 29, 3238.

(16) Compound $\mathbf{2 g}$ has not successfully undergone successful Pauson-Khand reactions in preliminary experiments. For rare examples using the acetamide tether, see: (a) Clive, D. L. J.; Cole, D. C.; Tao, Y. J. Org. Chem. 1994, 59, 1396; (b) Areces, P.; Durán, M. Á.; Plumet, J.; Hursthouse, M. B.; Light, M. E. J. Org. Chem. 2002, 67, 3506. For a reductive version, see: (c) Becker, D. P.; Flynn, D. L. Tetrahedron Lett. 1993, 34, 2087.

(17) (a) Sugihara, T.; Yamada, M.; Ban, H.; Yamaguchi, M.; Kaneko, C. Angew. Chem. Int. Ed. Engl. 1997, 36, 2801; (b) Sugihara, T.; Yamaguchi, M.; Nishizawa, M. Chem.Eur. J. 2001, 7, 1589.

(18) Sugihara, T.; Yamada, M.; Yamaguchi, M.; Nishizawa, M. Synlett 1999, 771.

(19) Experimental Procedure: A solution of $\mathbf{2 b}(73.0 \mathrm{mg}, 0.16$ $\mathrm{mmol}$ ) and 3.5 equivalents of cyclohexylamine (3.5 equiv, $64 \mu \mathrm{L})$ in 1,2-dichloroethane $(8 \mathrm{~mL})$ was heated to reflux for 20 minutes. The solution was cooled to room temperature, $20 \mathrm{~mL}$ diethyl ether $(20 \mathrm{~mL})$ was added, and the mixture was filtered through a pad of silica gel. Preparative TLC ( $4: 1$ petroleum ether:diethyl ether) of the crude material afforded $\mathbf{5 b}(20.2 \mathrm{mg}, 65 \%$ yield, 3.8:1 cis:trans).

(20) (5a) IR (neat, NaCl) $v_{\max } 3079,2957,1737,1715 \mathrm{~cm}^{-1} ;{ }^{1} \mathrm{H}$ NMR $\delta 5.84(\mathrm{~m}, 1 \mathrm{H}), 5.80(\mathrm{~m}, 1 \mathrm{H}), 4.69(\mathrm{br} \mathrm{d}, \mathrm{J}=10.9$, $1 \mathrm{H}), 4.28$ (apparent t, $\mathrm{J}=7.1,1 \mathrm{H}), 3.31(\mathrm{~m}, 1 \mathrm{H}), 3.29(\mathrm{~d}, \mathrm{~J}$ $=7.1,1 \mathrm{H}), 2.95-3.08(\mathrm{~m}, 2 \mathrm{H}), 2.63-2.71(\mathrm{~m}, 2 \mathrm{H}), 2.23(\mathrm{~m}$, $1 \mathrm{H}), 2.16(\mathrm{dd}, \mathrm{J}=18.5,2.7,1 \mathrm{H})$; resonances for the minor diastereomer were observed at 4.72 (apparent $\mathrm{t}, \mathrm{J}=6.0$, $1 \mathrm{H}$ ), 4.38 (apparent $\mathrm{t}, \mathrm{J}=7.4,1 \mathrm{H}), 3.46(\mathrm{dd}, \mathrm{J}=10.9,8.7$, $1 \mathrm{H}), 2.11(\mathrm{~J}=18.0,2.7,1 \mathrm{H}) ;{ }^{13} \mathrm{C}$ NMR $208.1179 .9,128.7$, 125.3, 72.9, 70.5, 43.2, 38.8, 33.3, 29.7, 25.1; MS m/e 176 $\left(\mathrm{M}^{+}\right)$; HRMS m/e for $\mathrm{C}_{11} \mathrm{H}_{12} \mathrm{O}_{2}$ calcd. $\left(\mathrm{M}^{+}\right)$176.0837, found 176.0842. (5b) IR (neat, $\mathrm{NaCl}$ ) $v_{\max } 3012,2922$, $1709,1678 \mathrm{~cm}^{-1} ;{ }^{1} \mathrm{H}$ NMR $\delta 5.59$, (ddd, $\mathrm{J}=12.4,7.2,2.1$, $1 \mathrm{H}), 5.61(\mathrm{~m}, 1 \mathrm{H}), 4.48(\mathrm{br} \mathrm{d}, \mathrm{J}=12.0,1 \mathrm{H}), 3.37(\mathrm{~m}, 1 \mathrm{H})$, $3.13(\mathrm{dd}, \mathrm{J}=10.9,6.7,1 \mathrm{H}), 3.07(\mathrm{~m}, 1 \mathrm{H}), 2.95(\mathrm{~m}, 1 \mathrm{H})$, $2.73(1 / 2$ ABquartet, $\mathrm{J}=6.3,1 \mathrm{H}), 2.70(1 / 2$ ABquartet, $\mathrm{J}=$ $6.3,1 \mathrm{H}),, 2.57(\mathrm{~m}, 1 \mathrm{H}), 2.43(\mathrm{~m}, 1 \mathrm{H}), 2.22(\mathrm{dd}, \mathrm{J}=17.1$, $4.11 \mathrm{H})$; resonances for the minor diastereomer were observed at $4.20(\mathrm{br} \mathrm{d}, \mathrm{J}=4.20,1 \mathrm{H}), 2.66(\mathrm{~d}, \mathrm{~J}=6.5,1 \mathrm{H})$, 2.18 (obscured dd, 1H) ${ }^{13} \mathrm{C}$ NMR 207.0, 183.4, 135.2, 126.6, 126.1, 47.9, 44.9, 42.6, 37.1, 32.3, 23.8; resonances for the minor diastereomer were observed at 129.2, 47.0, 43.2, $40.3 ; \mathrm{MS} \mathrm{m} / \mathrm{e} 192\left(\mathrm{M}^{+}\right)$; HRMS m/e for $\mathrm{C}_{11} \mathrm{H}_{14} \mathrm{O}_{2}$ calcd. $\left(\mathrm{M}^{+}\right)$192.0603, found 192.0580. (5c) IR (neat, $\mathrm{NaCl}$ ) $v_{\max } 2927,1714,1680 \mathrm{~cm}^{-1} ;{ }^{1}$ H NMR $\delta 4.74(\mathrm{dd}, \mathrm{J}=10.9$, 5.7, 1H), 4.27 (apparent t $\mathrm{J}=7.7 ., 1 \mathrm{H}), 3.36(\mathrm{dd}, \mathrm{J}=11.3$, $7.7,1 \mathrm{H}), 3.27(\mathrm{~m}, 1 \mathrm{H}), 2.64(\mathrm{dd}, \mathrm{J}=17.7,6.3,1 \mathrm{H}), 2.58$ $(\mathrm{m}, 1 \mathrm{H}), 2.21(\mathrm{~m}, 1 \mathrm{H}), 2.14(\mathrm{dd}, \mathrm{J}=17.6,3.1,1 \mathrm{H}), 2.09$ $(\mathrm{m}, 1 \mathrm{H}), 1.99(\mathrm{~m}, 1 \mathrm{H}), 1.93(\mathrm{~m}, 1 \mathrm{H}), 1.56(\mathrm{~m}, 1 \mathrm{H}), 1.30$ $1.49(\mathrm{~m}, 2 \mathrm{H})$; resonances for the minor diastereomer were observed at 4.35 (obscured $\mathrm{m}, 1 \mathrm{H}), 4.32$ (apparent $\mathrm{t}, \mathrm{J}=$
$7.8,1 \mathrm{H}), 3.35(\mathrm{dd}, \mathrm{J}=11.4,8.3,1 \mathrm{H}), 2.26(\mathrm{dd}, \mathrm{J}=16.6$, $4.7,1 \mathrm{H}), 2.55$ (obscured $\mathrm{m}, 1 \mathrm{H}) ;{ }^{13} \mathrm{C}$ NMR 208.4, 186.0, 137.7, 79.6, 71.9, 44.8, 41.0, 34.0, 27.6, 26.5, 23.5; resonances for the minor diastereomer were observed at 206.8 , 182.2, 135.4, 77.1, 71.4, 43.6, 39.0, 29.5, 27.5, 24.6, 23.4 MS m/e $178\left(\mathrm{M}^{+}\right), 150\left(\mathrm{M}-\mathrm{CO}^{+}\right)$; HRMS m/e for $\mathrm{C}_{11} \mathrm{H}_{14} \mathrm{O}_{2}$ calcd. $\left(\mathrm{M}-\mathrm{CO}^{+}\right)$150.1045, found 150.1038. (5d) IR (neat, $\mathrm{NaCl}) v_{\max } 2928,1713,1677 \mathrm{~cm}^{-1} ;{ }^{1}$ H NMR $\delta 4.79$ (ddd, J $=10.9,5.7,1.6,1 \mathrm{H}), 3.93(\mathrm{~d}, \mathrm{~J}=8.0,1 \mathrm{H}), 3.54(\mathrm{~d}, \mathrm{~J}=8.0$, 1H), 2.55 (apparent dt, $\mathrm{J}=16.1,4.2,1 \mathrm{H}), 2.39(1 / 2$ ABquartet, $\mathrm{J}=17.1,1 \mathrm{H}), 2.35$ (1/2 ABquartet, $\mathrm{J}=17.1$, $1 \mathrm{H}), 2.21(\mathrm{~m}, 1 \mathrm{H}), 2.05(\mathrm{~m}, 1 \mathrm{H}), 1.98(\mathrm{~m}, 1 \mathrm{H}), 1.90(\mathrm{~m}$, 1H), $1.55(\mathrm{~m}, 1 \mathrm{H}), 1.40(\mathrm{~m}, 1 \mathrm{H}), 1.33(\mathrm{~m}, 1 \mathrm{H}), 1.31(\mathrm{~s}$, 3H); ${ }^{13}$ C NMR 208.1, 185.7, 136.2, 76.1, 48.6, 47.4, 33.8, 27.5, 24.9, 24.5, 23.4; MS m/e $192\left(\mathrm{M}^{+}\right)$; HRMS m/e for $\mathrm{C}_{12} \mathrm{H}_{16} \mathrm{O}_{2}$ calcd. $\left(\mathrm{M}^{+}\right)$192.1150, found 192.1143. (5e) IR (neat, NaCl) $v_{\max } 2928,1703,1665 \mathrm{~cm}^{-1} ;{ }^{1} \mathrm{H}$ NMR $\delta 4.14$ (br d, J = 11.6, 1H), $3.31(\mathrm{~m}, 1 \mathrm{H}), 3.04(\mathrm{dd}, \mathrm{J}=10.6,6.7$, $1 \mathrm{H}), 2.69(\mathrm{dd}, \mathrm{J}=17.0,6.0,1 \mathrm{H}), 2.65(\mathrm{~m}, 1 \mathrm{H}), 2.60$ (apparent $\mathrm{t}, \mathrm{J}=11.3,1 \mathrm{H}), 2.18(\mathrm{dd}, \mathrm{J}=16.8,4.3,1 \mathrm{H}), 2.04$ (m, 1H), 1.85-1.97 (m, 2H), $1.53(\mathrm{~m}, 2 \mathrm{H}), 1.47(\mathrm{~m}, 1 \mathrm{H})$, $1.14(\mathrm{~m}, 1 \mathrm{H})$; resonances for the minor diastereomer were observed at $4.19(\mathrm{br} \mathrm{d}, \mathrm{J}=11.3,1 \mathrm{H}), 2.65(\mathrm{~J}=18.5,6.2$, $1 \mathrm{H}), 2.58(\mathrm{~m}, 1 \mathrm{H}), 2.23(\mathrm{~m}, 1 \mathrm{H}), 1.41(\mathrm{~m}, 1 \mathrm{H}) ;{ }^{13} \mathrm{C} \mathrm{NMR}$ 207.4, 184.9, 139.0, 48.5, 42.6, 36.1, 31.2, 30.9, 27.0, 23.0; resonances for the minor diastereomer were observed at 208.1, 181.0, 139.5, 48.2, 46.8, 41.2, 37.4, 35.3, 28.5, 23.5 MS m/e $194\left(\mathrm{M}^{+}\right)$; HRMS (ESI) m/e for $\mathrm{C}_{11} \mathrm{H}_{14} \mathrm{OS}$ calcd. $\left(\mathrm{M}+\mathrm{Na}^{+}\right) 217.0658$, found 217.0650. (5f) IR (neat, $\mathrm{NaCl}$ ) $v_{\max } 2928,1702,1648 \mathrm{~cm}^{-1} ;{ }^{1}$ H NMR $\delta 4.23$ (dd, J = 9.9, $4.2,1 \mathrm{H}), 4.06(\mathrm{~m}, 1 \mathrm{H}), 3.66$ (apparent dt, $\mathrm{J}=2.1,11.9$, $1 \mathrm{H}), 2.77(\mathrm{~m}, 1 \mathrm{H}), 2.62(\mathrm{dd}, \mathrm{J}=18.7,6.3,1 \mathrm{H}), 2.26-2.28$ $(2 \mathrm{H}), 1.97-2.15(\mathrm{~m}, 3 \mathrm{H}), 1.81-1.91(\mathrm{~m}, 2 \mathrm{H}), 1.28-1.75(\mathrm{~m}$, $4 \mathrm{H})$; resonances for the minor diastereomer were observed at 4.26 (br d, obscured, $1 \mathrm{H}), 4.09(\mathrm{~m}, 1 \mathrm{H}), 3.74(\mathrm{ddd}, \mathrm{J}=$ $11.4,9.3,4.6,1 \mathrm{H}), 3.01(\mathrm{~m}, 1 \mathrm{H}), 2.70(\mathrm{~m}, 1 \mathrm{H}), 2.67(\mathrm{dd}, \mathrm{J}$ $=18.2,6.3,1 \mathrm{H}), 2.70(\mathrm{~m}, 1 \mathrm{H}), 1.41(\mathrm{~m}, 1 \mathrm{H}), 1.14(\mathrm{~m}, 1 \mathrm{H})$; ${ }^{13} \mathrm{C}$ NMR 207.5, 170.4, 139.2, 78.6, 66.8, 41.0, 37.8, 34.0, $32.2,25.9,22.3,21.3$; resonances for the minor diastereomer were observed at $206.5,179.3,137.5,63.6$, 41.1, 32.3, 31.9, 28.2, 27.5, 26.6; MS m/e $192\left(\mathrm{M}^{+}\right)$; HRMS (ESI) $\mathrm{m} / \mathrm{e}$ for $\mathrm{C}_{11} \mathrm{H}_{14} \mathrm{O}_{2}$ calcd. $\left(\mathrm{M}+\mathrm{Na}^{+}\right)$215.1048, found 215.1050. (5i) IR (neat, $\mathrm{NaCl}$ ) $v_{\max } 2923,1714,1680$ $\mathrm{cm}^{-1} ;{ }^{1} \mathrm{H}$ NMR $\delta 7.78(\mathrm{~d}, \mathrm{~J}=8.2,2 \mathrm{H})$ and $7.72(\mathrm{~d}, \mathrm{~J}=8.2$, $2 \mathrm{H}), 7.34(\mathrm{~d}, \mathrm{~J}=8.2,2 \mathrm{H})$ and $7.38(\mathrm{~d}, \mathrm{~J}=8.2,2 \mathrm{H}), 4.46$ (ddd, $\mathrm{J}=10.9,5.4,1.51 \mathrm{H}), 4.00(\mathrm{dd}, \mathrm{J}=12.0,7.8,1 \mathrm{H})$, 3.96 (apparent $\mathrm{t}, \mathrm{J}=7.8,1 \mathrm{H}), 3.57(\mathrm{br} \mathrm{d}, \mathrm{J}=9.8,1 \mathrm{H}), 3.12$ $(\mathrm{m}, 1 \mathrm{H}), 2.94$ (apparent $\mathrm{t}, \mathrm{J}=11.5), 2.73(\mathrm{~m}, 1 \mathrm{H}), 2.62(\mathrm{dd}$, $\mathrm{J}=16.9,6.0,1 \mathrm{H}), 2.50-2.54(\mathrm{~m}, 3 \mathrm{H}), 2.43(\mathrm{~s}, 3 \mathrm{H})$ and 2.45 $(\mathrm{s}, 3 \mathrm{H}), 2.39-2.50(\mathrm{~m}, 3 \mathrm{H}), 2.18(\mathrm{~m}, 1 \mathrm{H}), 1.96-2.10(\mathrm{~m}$, $4 \mathrm{H}), 1.79-1.93(\mathrm{~m}, 2 \mathrm{H}), 1.43-1.64(\mathrm{~m}, 4 \mathrm{H}), 1.24-1.39(\mathrm{~m}$, 2H), $1.13(\mathrm{~m}, 1 \mathrm{H}) ;{ }^{13} \mathrm{C}$ NMR 207.2, 205.4, 180.9, 178.5, $144.3,143.8,139.2,137.7,136.0,131.7,130.0,129.9$, 128.0, 127.1, 62.6, 61.4, 54.2, 52.7, 41.6, 40.9, 40.4, 39.3, 35.7, 30.9, 30.3, 28.1, 27.1, 27.0, 25.9, 23.5, 22.8, 21.6; MS m/e $194\left(\mathrm{M}^{+}\right)$; HRMS m/e for $\mathrm{C}_{12} \mathrm{H}_{18} \mathrm{O}_{2}$ calcd. $\left(\mathrm{M}^{+}\right)$ 194.1301, found 194.1295.

(21) Rosillo, M.; Casarrubios, L.; Domínguez, G.; PérezCastells, J. Org. Biomol. Chem. 2003, 1, 1450. 
